\title{
The Memory Function of Noradrenergic Activity in Non-REM Sleep
}

\author{
Steffen Gais ${ }^{1,2}$, Björn Rasch ${ }^{1}$, Johannes C. Dahmen ${ }^{1}$, Susan Sara ${ }^{3}$, \\ and Jan Born ${ }^{4}$
}

\begin{abstract}
There is a long-standing assumption that low noradrenergic activity during sleep reflects mainly the low arousal during this brain state. Nevertheless, recent research has demonstrated that the locus coeruleus, which is the main source of cortical noradrenaline, displays discrete periods of intense firing during non-REM sleep, without any signs of awakening. This transient locus coeruleus activation during sleep seems to occur in response to preceding learning-related episodes. In the present study, we manipulate noradrenergic activity during sleep in
\end{abstract}

\section{INTRODUCTION}

New memories have to undergo a post-acquisition phase of consolidation in which they become stable and resistant to interference (McGaugh, 2000). In particular, the neuromodulator noradrenaline (NA) has been investigated as one of the factors contributing to memory consolidation (Sara, 2009; McGaugh, 2004; Paré, 2003). Recently, its contribution to emotional memory and especially its role in the development of traumatic memories have received much attention (Kindt, Soeter, \& Vervliet, 2009; de Quervain et al., 2007). The role of NA in memory function in general has been studied at the synaptic as well as at the systems level (van Stegeren, Wolf, Everaerd, \& Rombouts, 2008; Harley, 2007; Gibbs \& Summers, 2002; McGaugh, 2002). For instance, at the synaptic level, the expression of long-term potentiation (LTP) and long-term depression can both be modulated by NA release (Kemp \& Manahan-Vaughan, 2008; Hu et al., 2007; Kirkwood, Rozas, Kirkwood, Perez, \& Bear, 1999; Katsuki, Izumi, \& Zorumski, 1997; Bröcher, Artola, \& Singer, 1992). When looking at the systems level, the hippocampus and the amygdala appear to be the two regions especially sensitive to the effects of NA (Tully, Li, Tsvetkov, \& Bolshakov, 2007; Berlau \& McGaugh, 2006; Roozendaal, Hahn, Nathan, de Quervain, \& McGaugh, 2004). In particular, the interaction of these two regions seems to modulate synaptic plasticity (Vouimba, Yaniv, \& Richter-Levin, 2007; Akirav \& Richter-

${ }^{1}$ University of Lübeck, ${ }^{2}$ Ludwig Maximilians University of Munich, ${ }^{3}$ Collège de France, Paris, France, ${ }^{4}$ University of Tübingen humans with either the $\alpha 2$-autoreceptor agonist clonidine or the noradrenaline reuptake inhibitor reboxetine. We show that reducing noradrenergic activity during sleep, but not during wakefulness, impairs subsequent memory performance in an odor recognition task. Increasing noradrenergic availability during sleep, in contrast, enhances memory retention. We conclude that noradrenergic activity during non-REM sleep interacts with other sleep-related mechanisms to functionally contribute to off-line memory consolidation.

Levin, 2002). Importantly, NA can affect memory not only when present during encoding but also post-learning during the consolidation phase (McGaugh \& Roozendaal, 2002; Southwick et al., 2002).

Sleep provides a very specific context for brain activity, which is constituted essentially of reduced sensory interference and particular patterns of neuromodulatory activity, gene activity, and electrophysiological properties (Llinas \& Steriade, 2006; Pace-Schott \& Hobson, 2002; Cirelli \& Tononi, 2000). Many of these sleep-related changes have already been linked to aspects of memory function, thus establishing the idea of sleep as an ensemble of brain state modulations, which serve, among other purposes, the processing and consolidation of memories (Marshall \& Born, 2007; Born, Rasch, \& Gais, 2006; Frank \& Benington, 2006; Graves, Pack, \& Abel, 2001). During sleep, the pattern of NA release differs from that during wakefulness. Whereas other noradrenergic areas, for example, in the tegmentum, project to the brainstem and cranial nerves, the locus coeruleus (LC) is the source of noradrenalin in the neocortex and hippocampus (Aston-Jones, 2004; Gibbs \& Summers, 2002; Foote, Bloom, \& Aston-Jones, 1983). The LC fires tonically during quiet wakefulness and shows stimulus-related bursts during activity (AstonJones \& Cohen, 2005; Berridge \& Waterhouse, 2003). The tonic rate of firing correlates directly with the level of alertness displayed by the individual. During non-REM sleep, tonic LC activity decreases gradually with sleep depth. During REM sleep, LC neurons stop firing altogether (Pace-Schott \& Hobson, 2002; Aston-Jones \& Bloom, 1981). However, during phasic features of SWS, that is, 
sleep spindles and slow oscillations, LC neurons show burstfiring activity (Aston-Jones \& Bloom, 1981). In anesthetized rats, LC burst firing can be entrained by the frontal slow oscillation that characterizes SWS (Lestienne, Herve-Minvielle, Robinson, Briois, \& Sara, 1997). Moreover, recently, a specific window of strong LC activity during SWS around $120 \mathrm{~min}$ after learning periods has been discovered in rats (Eschenko \& Sara, 2008). Because sleep spindles and slow oscillations have been linked to memory consolidation and reprocessing during sleep (Ji \& Wilson, 2007; Marshall \& Born, 2007; Marshall, Helgadottir, Molle, \& Born, 2006; Gais, Mölle, Helms, \& Born, 2002), these findings together have led us to hypothesize that the burst activity of the LC noradrenergic system during SWS plays a specific role in the sleeprelated consolidation of recently acquired memories.

Here, we investigate the function of noradrenergic activity during non-REM sleep in healthy human subjects. To follow previous studies done in rats as closely as possible, we employ an odor recognition task, which has been shown to depend on delayed post-trial $\beta$-adrenergic receptor activation in rats (Sara, Roullet, \& Przybyslawski, 1999). We hypothesize that during periods of non-REM sleep, consolidation of previously learned odors would lead to enhanced odor recognition as compared with periods of wakefulness. Blockade of noradrenergic activity during this period with the $\alpha 2$-autoreceptor agonist clonidine should prevent sleep-related improvements. On the other hand, increased availability of NA by administration of the reuptake inhibitor reboxetine during sleep should improve consolidation of odor recognition memory.

\section{METHODS}

\section{Subjects and General Procedure}

Three groups of subjects participated in the current experiments. In Experiment 1 , a sleep $(n=14)$ and a sleep deprivation ( $n=14$ ) group were treated with the $\alpha 2$-noradrenergic autoreceptor agonist clonidine, systemic administration of which inhibits activity of LC neurons and blocks NA release from its synaptic terminals in the cortex (Washburn \& Moises, 1989). In Experiment 2, subjects $(n=16)$ received the NA reuptake inhibitor reboxetine, which increases extracellular NA (see Figure 1A,
Figure 1. Experimental design and main results (A) Subjects learned an odor recognition task and a word pair association task on the first evening (Le). Before sleep or sleep deprivation, they received clonidin $(\mathrm{C})$, reboxetine $(\mathrm{R})$, or placebo (P). Recall was tested later after the effects of the substances had ended (Re). (B) Number of correctly recognized odors in the clonidine (gray) and placebo conditions (white). Clonidine impaired memory consolidation only when administered during sleep but not during wakefulness. Subjects receiving clonidine during sleep displayed memory recall comparable with subjects from the wake group. (C) NA levels measured in peripheral blood in the placebo (solid) and clonidine conditions (dashed). Although the wake group (triangles) showed a numerically larger reduction in NA than the sleep group (circles), only the latter reached significance between 15 and 135 min after substance administration. Time is referenced to the

onset of substance administration. (D) Administration of the NA reuptake inhibitor reboxetine, which increases NA availability, during sleep enhances later memory recall (gray) compared with a placebo condition (white). Error bars represent SEM. * $p<.05$, **p $<.01$ for respective pairwise comparisons.

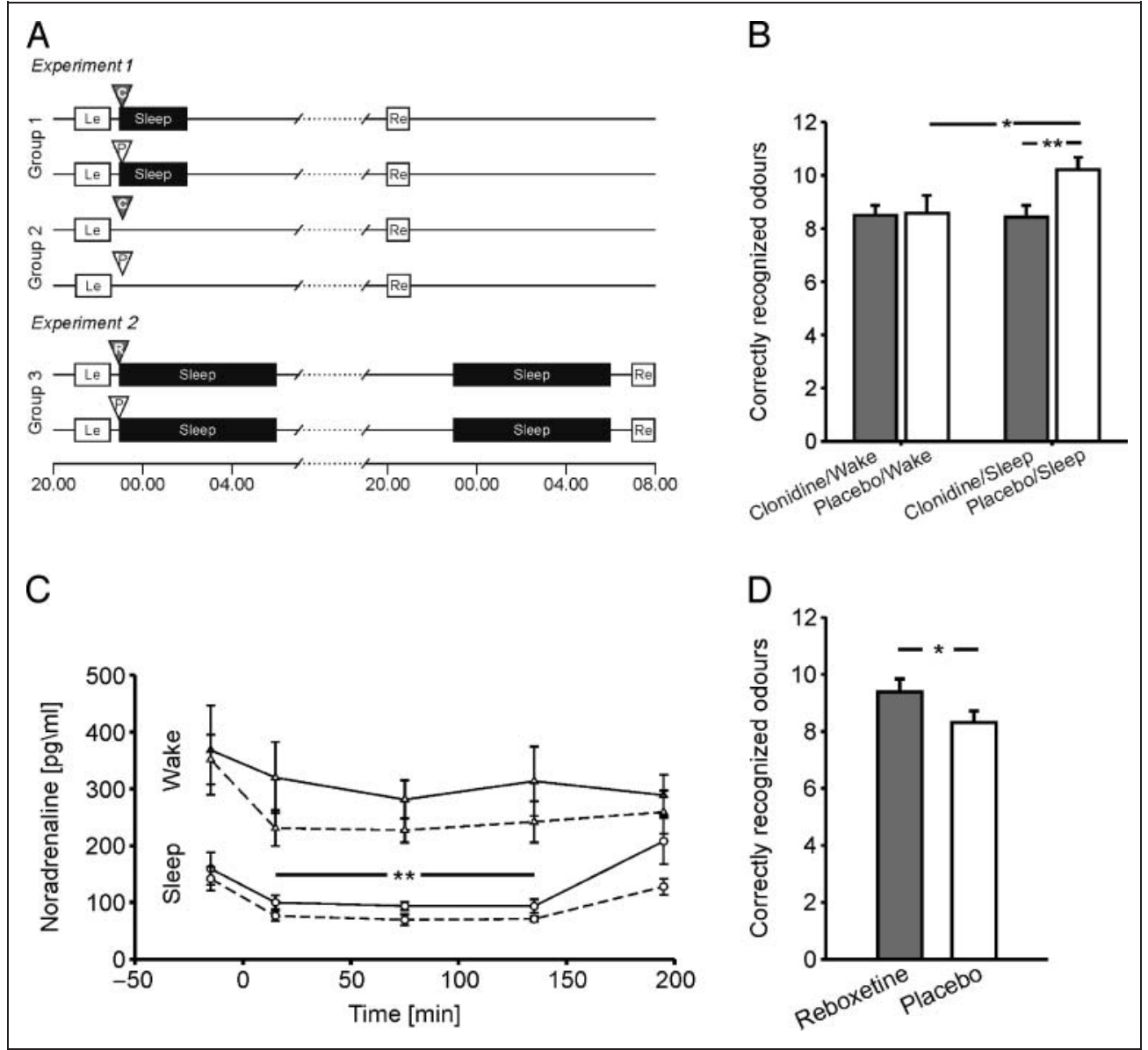


for an illustration of the study design). All subjects were between 18 and 35 years old (mean age $=25.7$ years, $S D=1.3$ years), male, and nonsmoking. Subjects were healthy, taking no medications, and having no neurologic or psychiatric disorders. They reported a normal sleepwake cycle. On experimental days, they were not allowed caffeine or alcohol, and sleeping was prohibited during daytime. Experiments were approved by the ethics committee of the University of Lübeck, and informed consent was obtained from all subjects.

Before the experiments, subjects were accustomed to sleeping under laboratory conditions during an adaptation night. Each subject participated in two experimental sessions (substance and placebo), separated by an interval of at least 2 weeks according to a within-subject, double-blind, cross-over design. The order of conditions was balanced across subjects. Sessions started at 20:30 hr with the placement of an intravenous catheter for substance administration and blood sampling. At 21:00 hr, the first presentation of an odor recognition task took place. At 21:30 hr, subjects learned a paired-associate word list task, followed at 22:00 hr by a second presentation of the odor recognition task. At 22:30 hr, electrodes were applied for polysomnography (EEG, EOG, and EMG). At 23:00 hr, subjects went to bed (Experiments 1 and 2, sleep groups) or they were sleep deprived (Experiment 1, clonidine, wake group). Subjects were awoken after at least $3 \mathrm{hr}$ (Experiment 1, clonidine, sleep group) or $7 \mathrm{hr}$ (Experiment 2, reboxetine) of polysomnographically verified sleep. Off-line scoring of sleep did not always agree with on-line detection; therefore, actual sleep durations in some subjects exceed the envisaged duration. For Experiment 1 , subjects then had to stay awake until the next evening when retention performance was tested at 20:00 hr. Note that, although the long wake period increased the subjects' fatigue at recall testing, this influence was equal for both the placebo and clonidine conditions. For Experiment 2, subjects stayed awake during the following day and slept at home on the following night before retention performance was tested at 07:00 hr on the second morning after learning. During nighttime wake periods, subjects were under constant surveillance by an experimenter and were allowed to play games and watch movies. Activity when not in the laboratory was monitored via actigraphy (Actiwatch, Cambridge Neurotechnology, Cambridge, UK).

\section{Odor Learning Task}

The odor learning task used in these experiments was designed to closely match learning tasks employed in experiments with rats to provide maximum comparability with these studies (Sara et al., 1999). Subjects had to sniff at flasks containing distinct, unknown odors. During learning, six odors were presented for $20 \mathrm{sec}$ each. Each odor then had to be rated on 5-point scales measuring familiarity (0 [completely unknown] to 4 [well known] $)$, intensity (0 [does not smell at all $]$ to 4 [smells very intensively]), and pleasantness ( -2 [very unpleasant] to 2 [very pleasant]). Additionally, subjects were asked whether they could detect and distinguish all odors. $30 \mathrm{~min}$ after the end of the first presentation; the whole learning procedure was repeated with the same odors. After the retention interval, subjects were given 12 odors, six old and six new ones. For each odor, they had to decide whether it had been presented previously or not. Time until decision was measured, and the subject was asked to give a confidence rating (0-4) on his decision. In addition, subjects had to judge intensity, pleasantness, and familiarity once more. On the second visit, a new set of 12 odors was used. Odors were randomly assigned to both sessions, and the order of presentation varied randomly.

Odors and their concentrations were taken from Sulmont, Issanchou, and Köster (2002). Twenty-four odors (see Table 1) were diluted in mineral oil to obtain an intensity similar to butan-1-ol at $0.2 \mathrm{ml} / \mathrm{L}$. Each substance $(12.5 \mathrm{ml})$ was given into a $60-\mathrm{ml}$ sealable, brown flask containing an $11 \times 5.5 \mathrm{~cm}$ piece of absorbent tissue (Chemical Absorbent Sheets Type P110, 3M, Neuss, Germany). Flasks were stored at $5^{\circ} \mathrm{C}$ until $1 \mathrm{hr}$ before the beginning of the experiments. To smell the odors, subjects took each flask and held it close to their noses $(1-3 \mathrm{~cm})$. There was a 1-min ISI to avoid olfactory adaptation.

\section{Word List Learning Task}

Subjects were presented with a list of 40 semantically related German word pairs, which were nonemotional nouns, representing concrete objects. Subjects had to learn the word pairs to a criterion of at least $60 \%$. The word pairs were presented sequentially on a computer screen for $5 \mathrm{sec}$ each, with an intertrial interval of $100 \mathrm{msec}$. After presentation of the complete list, cued recall was tested immediately. The first word of each pair was presented, and the subject had to name the second one. Afterwards, the correct answer was displayed for $2 \mathrm{sec}$, thus allowing the subject to correct his memory if necessary. If the $60 \%$ criterion was not reached, recall testing was repeated. After the retention interval, cued recall was tested again.

\section{Substance Administration}

In Experiment 1, subjects received an intravenous infusion of $112.5 \mu \mathrm{g}$ of the $\alpha 2$-noradrenergic autoreceptor agonist clonidine (Catapresan, Boehringer Ingelheim Pharma, Ridgefield, CT), which passes the blood-brain barrier and suppresses activity of the LC (Washburn \& Moises, 1989). The infusion was administered via a forearm catheter. The substance was diluted in $17 \mathrm{ml}$ of physiologic saline solution and infused over 10 min starting from sleep onset (first occurrence of sleep stage 2). According to the cross-over design of the experiments, subjects received $17 \mathrm{ml}$ of physiologic saline solution as a placebo 
in the other condition. In Experiment 2, subjects received $2 \mathrm{mg}$ reboxetine (Edronax, Pfizer, New York, NY) in form of capsules or identically looking placebo capsules with a glass of water directly before going to sleep. The drugs used have half-lives of $7.5 \mathrm{hr}$ (clonidine) and 13 hr (reboxetine) (Dostert, Benedetti, \& Poggesi, 1997; Keranen, Nykanen, \& Taskinen, 1978). To exclude substance effects on retrieval testing (instead of on consolidation), the experimental design included the following features: First, retrieval testing was timed in a way that the fraction of substance remaining unmetabolized in the system was marginal. Therefore, retention tests took place $24 \mathrm{hr}$ (clonidine, Experiment 1) and $34 \mathrm{hr}$ (reboxetine, Experiment 2) after initial learning. Second, we can exclude effects on testing by means of the wake control group. If the wake group does not show an effect of clonidine, any effect observed in the sleep group cannot be due to a simple direct action of the substance on testing.

\section{Blood Sampling}

An intravenous catheter was placed in the forearm of the subject. Via long plastic tubes passing through the wall of the sleeping chamber, substance administration and blood sampling could be performed without the subject noticing and without disturbing the subject's sleep. Blood samples were taken at lights off and then hourly, starting 15 min after sleep onset and finally 15 min after awakening. Blood samples were immediately centrifuged, and plasma and serum were stored at $-20^{\circ} \mathrm{C}$ until assay. Because, although the mechanism is still tentative, there is a stable correlation between peripheral and central aminergic levels (Rasch, Dodt, Molle, \& Born, 2007; Williams \& McGaugh, 1993; Gold \& van Buskirk, 1978), peripheral NA levels were determined from EDTA-plasma by highperformance liquid chromatography (Waters, Milford, MA) with electrochemical detection. Cortisol was measured from serum using an enzyme-linked immunosorbent assay (Enzymun-Test Cortisol, Roche Diagnostics, Mannheim, Germany). Growth hormone was assessed from serum by competitive chemoluminescence immunoassay with an Immulite System (DPC-Biermann GmbH, Bad Nauheim, Germany). Additionally, one drop of blood was used to immediately determine levels of blood glucose with a HemoCue Glucose System (HemoCue GmbH, Grossostheim, Germany).

\section{Sleep Recordings}

Sleep was recorded polysomnographically by means of EEG at positions C3 and C4, EOG, and EMG and scored according to standard criteria by two experienced scorers. Wakefulness during the nights was assured by the permanent presence of an experimenter. Wakefulness during daytime was verified by actimetry (Actiwatch, Cambridge Neurotechnology, Cambridge, UK). Additionally, blood pressure was measured regularly from the outside with a cuff connected via 2-m tubes to an automatic device (boso-medicus prestige, Bosch + Sohn GmbH, Juningen, Germany). Because subjects were accustomed to this procedure during the adaptation nights, it did not disturb their sleep. Blood pressure was measured at lights off, at the beginning of infusion and 15 and 30 min thereafter, and then every $30 \mathrm{~min}$ until $1 \mathrm{hr}$ after awakening.

\section{Statistical Analysis}

All results are given as means $\pm S E M$. Statistical testing was done using paired $t$ tests. The interaction between sleep-wakefulness and placebo-substance was tested with a two-factorial ANOVA. Results were considered significant if a probability of $p<.05$ (two tailed) was reached. $d^{\prime}$ values were calculated for recognition measures. A standard correction was applied for hit rates of $100 \%$ [replace by $1-1 /(2 N)$ ] and false alarm rates of $0 \%$ [replace by $1 /(2 N)$ ]. In Experiment 2, three subjects were excluded from the analysis because they spent more than $30 \%$ of the night awake or in light sleep Stage 1.

\section{RESULTS}

\section{Effects of Noradrenergic Suppression by Clonidine}

In Experiment 1, noradrenergic activity was modulated by administration of clonidine, which is an agonist at $\alpha 2$-autoreceptors and known to suppress central noradrenergic activity (Langer, 1980). Subjects who slept during a 3-hr period with high percentage of SWS after learning showed significantly enhanced memory for odor stimuli compared with waking subjects (correctly identified odors: $10.2 \pm 0.5$ and $8.6 \pm 0.7, d^{\prime}=1.9 \pm 0.2$ and $1.2 \pm 0.2$, for placebo conditions of sleep and wake groups, respectively; $t_{26}=2.1, p<.05$, effect size $d=0.8$; Figure $1 \mathrm{~B}$ ), confirming an effect of sleep on the consolidation of this kind of memory task. When subjects received clonidine during sleep after learning, the sleep-related enhancements in odor memory performance were completely eliminated compared with the placebo condition (correctly identified odors: $8.4 \pm 0.4$ and $10.2 \pm 0.5, d^{\prime}=1.1 \pm 0.2$ and $1.9 \pm$ 0.2 , for clonidine and placebo, respectively; $t_{13}=-3.3$, $p<.01$, effect size $d=-1.0$; Figure 2). Response time did not differ significantly between placebo and substance conditions $(5.8 \pm 1.0$ and $6.1 \pm 0.8$ for clonidine and placebo, respectively; $t_{13}=0.4, p=.7$ ). Confidence in odor memory judgments also did not differ significantly between conditions $(2.8 \pm 0.1$ and $2.9 \pm 0.2$ for clonidine and placebo, respectively; $\left.t_{13}=0.2, p=.8\right)$. On the other hand, when subjects received clonidine during a period of wakefulness after learning, clonidine had no effect on memory performance (correctly identified odors: $8.5 \pm$ 0.4 and $8.6 \pm 0.7, d^{\prime}=1.2 \pm 0.3$ and $1.2 \pm 0.2$, for clonidine and placebo, respectively; $\left.t_{13}=0.3, p=.75\right)$. This difference between the effect of clonidine administered during sleep and during wakefulness was confirmed by a 


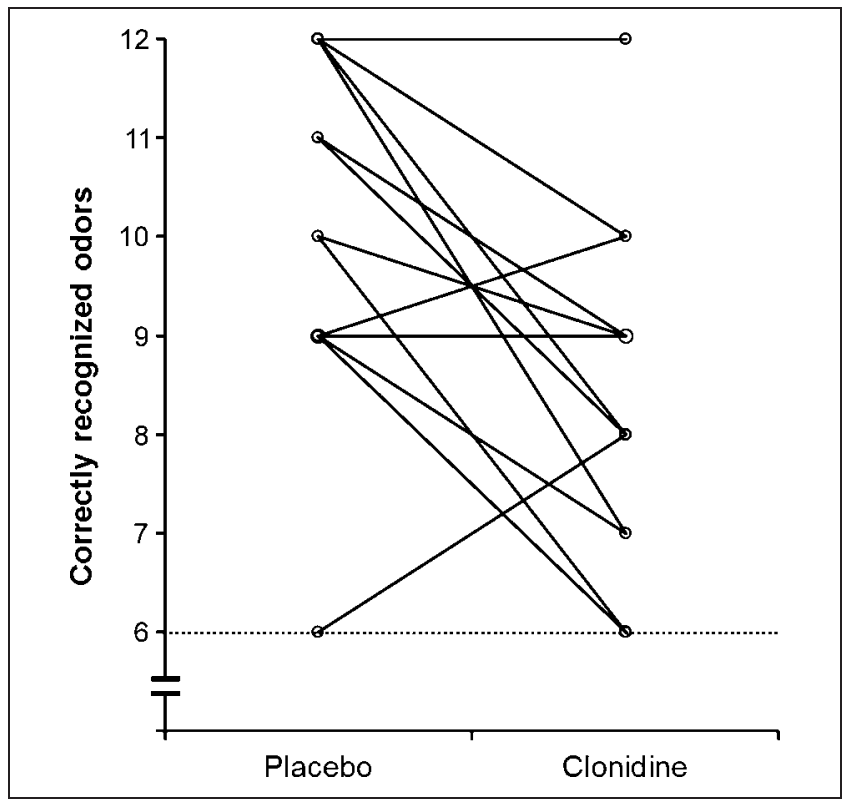

Figure 2. Distribution of individual data for the sleep group of Experiment 1 . Note that only two subjects with the lowest performance in the placebo condition show better performance in the clonidine condition. Thus, the negative influence of NA suppression by clonidine on odor memory consolidation during sleep seems to be stable across subjects. The dotted line represents chance performance.

significant interaction between the factors Sleep-wake $\times$ Placebo-clonidine $\left(F_{1,26}=4.8, p<.05\right)$.

In contrast to odor memory, memory for word pairs was not affected by clonidine treatment in either the sleep group or the wake group ( $p>.25$; Table 1$)$. Together, our results indicate an enhanced consolidation of odor memories during sleep, which is blocked if non-REM sleep-associated noradrenergic activity is suppressed by clonidine.

During the learning phase, odors were rated for intensity, on average, at $2.1 \pm 0.05$ (on a scale of $0-4$ ) and for pleasantness at $0.3 \pm 0.06$ (on a scale of -2 to 2 ). Thus, as intended, odors had a medium intensity and were neither pleasant nor unpleasant. There was no significant difference in error rates for old and new items $(0.16 \pm 0.06$ vs. $0.21 \pm 0.05)$. Polysomnographic recordings confirmed the expected prevalence of SWS during the 3-hr sleep period (Table 2). Sleep was unchanged by clonidine administration, apart from a slight reduction in REM sleep (clonidine: $6.0 \pm 1.6 \%$, placebo: $8.8 \pm 1.7 \% ; p<.05)$. There was no correlation between REM sleep duration and odor recognition or the change in REM sleep duration and the change in odor recognition between clonidine and placebo condition (both $r<0.02$ ) (Table 3).

Peripheral levels of NA reflected the sleep-wake state and the NA suppressing action of clonidine. Under placebo conditions, sleeping subjects showed considerably lower levels of NA than subjects who were awake (sleep: $97.5 \pm$ $9.5 \mathrm{pg} / \mathrm{ml}$, wake: $295.6 \pm 45.2 \mathrm{pg} / \mathrm{ml} ; t_{22}=4.0, p<.001$; Figure 1C). In the sleep group, clonidine significantly suppressed NA between 15 and 130 min after administration (clonidine: $70.1 \pm 6.7 \mathrm{pg} / \mathrm{ml}$, placebo: $97.5 \pm 9.5 \mathrm{pg} / \mathrm{ml}$; $\left.t_{12}=2.4, p<.05\right)$. In the wake group, decreased NA levels are also apparent (clonidine: $240.5 \pm 27.5 \mathrm{pg} / \mathrm{ml}$, placebo: $295.6 \pm 45.2 \mathrm{pg} / \mathrm{ml}$ ). However, although numerically much larger than during sleep, differences did not reach significance because of the large variability $(p>.30)$. Before substance administration, levels of NA did not differ between conditions ( $p>.60$ for sleep and wake groups).

Under placebo conditions, average blood pressure was significantly lower during sleep than during wakefulness (systolic blood pressure-sleep: $114 \pm 1.8$, wake: $126 \pm$ 2.9; diastolic blood pressure-sleep: $65 \pm 1.0$, wake: $80 \pm$ 2.6; both $p<.01$; Figure 3). During wakefulness, clonidine lowered blood pressure during an interval between 120 and 210 min after administration (systolic blood pressure-clonidine: $116 \pm 2.0$, placebo: $126 \pm 2.9$; diastolic blood pressure-clonidine: $74 \pm 1.6$, placebo: $80 \pm 2.6$; both $p<.01)$. No such reduction in blood pressure by clonidine

Table 1. List of Odorants with Unfamiliar Odor (from Sulmont et al., 2002).

2,6-Dimethylhept-5-enal

4-Phenylbutan-2-one

Adoxal (2,6,10-trimethyl undec-9-enal)

Butan-1-ol

Cyclabute (4,7-methano-2-methyl-3a,4,5,6,7,7a-hexahydro-(1H)inden-5-yl propanoate)

Damascenone

Decan-2-one

Heptan-2-one

Hex-2-enal

Lilestralis (4-tertiobutyl-2-methyl benzenepropanal)

Linalool oxyde

Octan-1-ol

Tridec-2-enenitrile (Ozonil)

Tridecan-2-one

Bornyl acetate

Menthyl acetate

Diethyl malonate

Methyl benzoate

Dec-9-en-1-ol

3,7-Dimethyloctanenitrile

Isobutyl quinoline

Vigoflor (perhydro spiro[2-furane-2, $5^{\prime}$-( $4^{\prime}, 7^{\prime}$-methano)indene])

Herbac (1-acetyl-3,3-dimethyl cyclohexane)

1-Phenylethyl acetate 
Table 2. Results of the Word Pair Learning Task

\begin{tabular}{|c|c|c|c|c|c|c|}
\hline & \multicolumn{2}{|c|}{ Experiment 1 (Sleep) } & \multicolumn{2}{|c|}{ Experiment 1 (Wake) } & \multicolumn{2}{|c|}{ Experiment 2 (Sleep) } \\
\hline & Clonidine & Placebo & Clonidine & Placebo & Reboxetine & Placebo \\
\hline Recall (pre) & $31.3 \pm 1.5$ & $33.1 \pm 1.2$ & $32.3 \pm 1.2$ & $31.5 \pm 1.1$ & $27.2 \pm 0.9$ & $28.5 \pm 0.7$ \\
\hline Recall (post) & $32.8 \pm 1.4$ & $33.8 \pm 1.0$ & $32.9 \pm 1.1$ & $30.7 \pm 1.0$ & $27.8 \pm 0.9$ & $28.3 \pm 0.9$ \\
\hline \multirow[t]{2}{*}{ Change } & $1.5 \pm 0.9$ & $0.8 \pm 0.9$ & $0.6 \pm 0.8$ & $-0.8 \pm 0.8$ & $+0.6 \pm 0.9$ & $-0.2 \pm 1.0$ \\
\hline & \multicolumn{2}{|c|}{$p>.25$} & \multicolumn{2}{|c|}{$p>.25$} & \multicolumn{2}{|c|}{$p>.24$} \\
\hline
\end{tabular}

Manipulation of noradrenergic activity during sleep or wakefulness did not have an effect on consolidation of nonemotional word pair memory. (Word pair data for Experiment 2 has been reported in Rasch, Pommer, Diekelmann, \& Born, 2008.)

was found in sleeping subjects (systolic blood pressureclonidine: $113 \pm 2.7$, placebo: $114 \pm 1.8$; diastolic blood pressure-clonidine: $65 \pm 1.5$, placebo: $65 \pm 1.0$; both $p>$.75). Levels of cortisol during sleep were closely comparable between clonidine and placebo conditions $(p>$ .45 , for all time points).

\section{Effects of Noradrenergic Augmentation by Reboxetine}

In a second experiment, we tested the effect of increasing noradrenergic availability by reboxetine administration during sleep. Because of the longer plasma half-life of reboxetine, a longer retention interval of $34 \mathrm{hr}$ (instead of $24 \mathrm{hr}$ ) was used including two 7-hr nocturnal periods of sleep. Therefore, as there is continuous forgetting of memory over time, slightly lower retention performance was expected than in the first experiment, which was employing a shorter retention interval. Subjects remembered, on average, $8.3 \pm 0.4$ odors after placebo, but $9.4 \pm$ 0.5 odors after reboxetine administration $\left(d^{\prime}=1.1 \pm 0.2\right.$ and $1.6 \pm 0.2$, respectively; $t_{12}=2.2, p<.05$, effect size $d=0.7$; Figure 1D). Thus, in contrast to clonidine, which decreased NA availability and impaired memory consolidation, NA reuptake inhibition by reboxetine enhanced odor memory consolidation.

In contrast to clonidine, reboxetine strongly disturbed sleep. Proportions of wakefulness and light sleep Stages $1+$
2 were significantly increased, whereas the occurrence of REM sleep was nearly totally blocked (Table 1). During the second night (recovery sleep) before retrieval testing, total sleep time as controlled by actigraphy did not differ between reboxetine and placebo conditions (reboxetine: $408 \pm 9 \mathrm{~min}$, placebo: $422 \pm 9 \mathrm{~min} ; p>$ .20). Both actions of reboxetine, the enhancement of memory consolidation and the reduction of REM sleep, were correlated $(r=-.66, p=.01)$. As with clonidine administration, treatment with reboxetine did not affect memory for word pairs $(p>.24$; Table 1$)$.

Peripheral levels of NA were unaltered by reboxetine (reboxetine: $84.9 \pm 8.3 \mathrm{pg} / \mathrm{ml}$, placebo: $88.4 \pm 9.9 \mathrm{pg} /$ $\left.\mathrm{ml} ; t_{7}=-0.3, p>.75\right)$. However, reboxetine strongly raises average cortisol levels during the first night of sleep $(10.95 \pm 1.71 \mu \mathrm{g} / \mathrm{dl})$ compared with placebo administration $(3.76 \pm 0.62 \mu \mathrm{g} / \mathrm{dl} ; p<.01)$. Levels of cortisol and NA were again comparable between experimental conditions at retrieval testing (all $p>.10$ ).

\section{DISCUSSION}

The inhibition of noradrenergic activity during sleep by administration of the $\alpha 2$-noradrenergic autoreceptor agonist clonidine suppressed sleep-induced enhancement of odor memory consolidation. In contrast, administration of the NA reuptake inhibitor reboxetine during sleep improved odor memory consolidation compared

Table 3. Percentage of Total Sleep Time Spent in Different Sleep Stages after Administration of Clonidine and Reboxetine versus Placebo, Respectively

\begin{tabular}{|c|c|c|c|c|c|c|}
\hline & \multicolumn{3}{|c|}{ Experiment 1 (3-hr Sleep) } & \multicolumn{3}{|c|}{ Experiment 2 (7-hr Sleep) } \\
\hline & Clonidine & Placebo & $p$ & Reboxetine & Placebo & $p$ \\
\hline Wake & $1.1 \pm 0.4 \%$ & $1.2 \pm 0.5 \%$ & & $8.1 \pm 0.7 \%$ & $4.1 \pm 1.1 \%$ & $<.01$ \\
\hline S1 & $8.4 \pm 2.4 \%$ & $7.9 \pm 2.2 \%$ & & $10.8 \pm 1.0 \%$ & $7.8 \pm 0.9 \%$ & \\
\hline S2 & $51.6 \pm 2.7 \%$ & $49.5 \pm 2.9 \%$ & & $64.5 \pm 1.6 \%$ & $55.3 \pm 1.9 \%$ & $<.001$ \\
\hline SWS & $32.9 \pm 3.2 \%$ & $32.5 \pm 3.4 \%$ & & $13.3 \pm 1.4 \%$ & $15.2 \pm 1.7 \%$ & \\
\hline REM & $6.0 \pm 1.6 \%$ & $8.8 \pm 1.7 \%$ & $<.05$ & $2.6 \pm 0.7 \%$ & $16.8 \pm 1.7 \%$ & $<.001$ \\
\hline Total Sleep Time & $199.1 \pm 4.5 \mathrm{~min}$ & $197.2 \pm 4.8 \mathrm{~min}$ & & $413.5 \pm 10.1 \mathrm{~min}$ & $422.3 \pm 8.8 \mathrm{~min}$ & \\
\hline
\end{tabular}




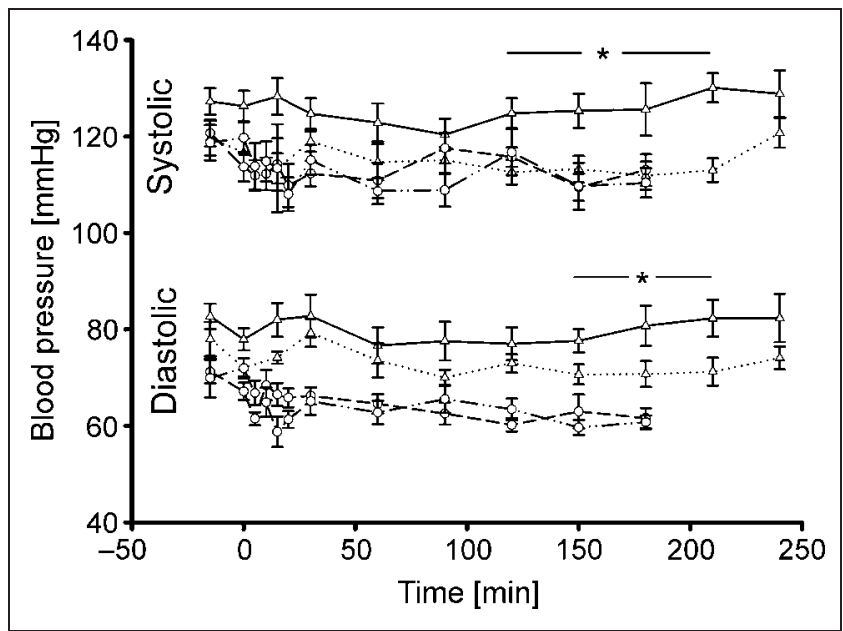

Figure 3. Blood pressure before and after administration of the $\alpha 2$-autoreceptor agonist clonidine. Between 120 and $210 \mathrm{~min}$ after substance administration, clonidine decreased systolic and diastolic blood pressure in the wake group (dotted) compared with the placebo condition (solid). In the sleep group, clonidine (dash-dotted) had no effect on blood pressure compared with placebo (dashed). Time is referenced to the onset of substance administration. Error bars represent SEM. * $p<.05$ for pairwise comparisons at single time points.

with a placebo condition. No effect of NA manipulation on memory consolidation was found during wakefulness, nor did NA manipulation affect memory for nonemotional word pair lists. Although possible differences in sleep and odor processing between humans and rats complicate straightforward comparisons, we designed the odor experiments to closely match experiments performed in rats testing the effects of $\beta$-adrenergic receptor blockade on a similar odor recognition task (Sara et al., 1999). That particular study showed that noradrenergic activity during a window around $2 \mathrm{hr}$ after learning was essential for memory consolidation. There, sleep was not examined. However, in another recent study, it was demonstrated that SWS-related noradrenergic LC burst activity increases during the same 2-hr post-learning time window (Eschenko \& Sara, 2008). Our results in human subjects, thus, confirm the prediction that non-REM sleeprelated noradrenergic activity during the first $3 \mathrm{hr}$ of sleep after learning is important for memory consolidation. Thus, whereas the global reduction of NA during SWS might support processes of synaptic downscaling (Tononi \& Cirelli, 2006), short, restricted noradrenergic bursts during this sleep stage might have the opposite function, specifically enhancing memory consolidation.

Our data show the expected supportive influence of sleep on the consolidation of odor memories in humans. The effect appeared after a short, 3-hr period of nocturnal sleep dominated by SWS, pointing once more to a possible relevance of this sleep stage for memory consolidation (Marshall \& Born, 2007). When viewing the present results together with previous studies, a characteristic triad of SWS-related activity appears to be of particular importance: the slow oscillation, the sleep spindle, and the
LC activity. With regard to the former two phenomena, studies in rodents and humans have yielded remarkably consistent findings (Mölle, Eschenko, Gais, Sara, \& Born, 2009; Steriade, 2003). Multiunit recordings in anesthetized rats reveal LC activity to be synchronized to the slow oscillation in the frontal cortex, with LC firing following the slow oscillation upstate by $\sim 300 \mathrm{msec}$ (Lestienne et al., 1997). Furthermore, LC activity is also associated with sleep spindle activity (Aston-Jones \& Bloom, 1981), and spindle activity, in turn, is grouped by the slow oscillation (Mölle, Marshall, Gais, \& Born, 2002). Sleep spindles, slow oscillation, and LC activity have all been individually linked to memory consolidation (Marshall et al., 2006; Gais et al., 2002), but their interaction might be of special importance. Especially the timing provided by the slow oscillation might allow the plasticity-enhancing actions of spindle-induced calcium influx and noradrenergic LC activity to act synergistically (Rosanova \& Ulrich, 2005; Stanton \& Sarvey, 1985). REM sleep, in contrast, does not appear to contribute to the consolidation of odor memories. Although reboxetine, consistent with previous findings, blocked REM sleep (Mayers \& Baldwin, 2005), odor memories were even improved as compared with the placebo condition.

There are multiple ways, direct or indirect, by which NA can influence memory function. NA can enhance memory by increasing general arousal and neuronal signalto-noise ratio (Aston-Jones \& Cohen, 2005; Gibbs \& Summers, 2002). It can also modulate memory encoding and consolidation through pathways from the basolateral amygdala to the enthorinal cortex, hippocampus, IC, or anterior cingulate (Roozendaal, McEwen, \& Chattarji, 2009; McGaugh, 2004; Paré, 2003; McGaugh \& Roozendaal, 2002). Although the amygdala is mainly involved in emotional processing and most memory effects of NA have been investigated with emotional stimuli, odor recognition is also known to recruit the amygdala (Savic, Gulyas, Larsson, \& Roland, 2000). On the other hand, the effects of NA are not restricted to the amygdala but influence other neocortical and hippocampal areas as well, where interaction with other transmitters, for example, acetylcholine, might come into play (Ramos \& Arnsten, 2007; Tzavara et al., 2006; Tronel, Feenstra, \& Sara, 2004; Miller, Wong, Chesnut, \& Dwoskin, 2002; Izquierdo et al., 1998; Bear \& Singer, 1986). At the synaptic level, these effects of NA might be related to its ability to stabilize early LTP and transform it into late LTP (Frey, Bergado-Rosado, Seidenbecher, Pape, \& Frey, 2001). Thus, for the present odor recognition task, both direct synaptic effects and a modulatory influence of NA within the amygdala are plausible.

Some other, more indirect effects of NA on memory consolidation are also conceivable. A change in cortisol levels can influence memory consolidation during sleep and wakefulness (McGaugh, 2004; Plihal \& Born, 1999). Notably, glucocorticoid-related enhancement of emotional memory requires NA activity in the basolateral amygdala. Thus, it is possible that odor memory enhancement through increased NA in the reboxetine experiment is 
amplified by cortisol release, which, in turn, potentiates the NA signaling cascade in the amygdala (Roozendaal et al., 2009). Clonidine, on the other hand, does not affect cortisol release and, thus, strengthens the conclusion that the observed modulation of memory requires NA activity. Sleep duration can also be excluded as a likely mediator of noradrenergic effects in our studies. In the clonidine experiments, the percentage of REM sleep was slightly reduced. However, reboxetine administration reduced the percentage of REM sleep, as well, but produced an opposite effect on memory consolidation. In addition, there is no correlation at all between REM sleep and odor memory performance in the clonidine experiments.

Memory can be impaired by blocking $\beta$-adrenergic receptors during the encoding phase (Cahill, Prins, Weber, \& McGaugh, 1994), directly afterwards (Cahill, Pham, \& Setlow, 2000) or 60 min after a memory trace has been reactivated by a single trial (Roullet \& Sara, 1998). These findings suggest that there are several windows of noradrenergic activity after learning which are essential for memory consolidation. In one-trial avoidance learning in chicks, at least two phases have been identified during the first 30 min after learning during which the memory traces are still labile and dependent on noradrenergic activity. When $\alpha 1$-adrenergic receptors are activated 5 or 25 min after learning by agonists or by very high levels of NA, memory transfer to long-term memory is prevented (Gibbs \& Summers, 2002). On the other hand, activation of $\beta$-receptors during the first $30 \mathrm{~min}$ after learning enhances memory consolidation (Gibbs \& Summers, 2002). In the rat, blockade of $\beta$-receptors 120 min after learning reveals another window of endogenous noradrenergic activation contributing to memory consolidation (Tronel et al., 2004; Sara et al., 1999). Additionally, it has been shown recently that there is a transient increase in noradrenergic LC firing activity during the same time window 120 min after learning, occurring only during SWS (Eschenko \& Sara, 2008). Whether these windows are similar in rats and humans and whether the relation to sleep is similar in these two species are still unknown. The present results speak for a delayed window of noradrenergic activity within the first $3 \mathrm{hr}$ of sleep after learning. They do not exclude a-qualitatively differentcontribution of NA during the first 30 min of wakefulness after learning, which have been spared in our experimental design because they might pertain mainly to early rather than late processes of LTP.

Declarative, hippocampus-dependent memory consolidation was not affected by manipulation of noradrenergic availability in the present experiment, suggesting that sleep can affect memory consolidation by different mechanisms, depending on the type of memory system involved. Memory for nonemotional words involves primarily occipital visual and medial prefrontal cortical areas and the hippocampus; consolidation presumably relies on an information transfer between the hippocampus and the neocortex (Gais et al., 2007; Hasselmo, 1999). Odor recognition, on the other hand, recruits mainly the piriform cortex, OFC, and amygdala (Plailly et al., 2005; Sanchez-Andrade, James, \& Kendrick, 2005; Buchanan, Tranel, \& Adolphs, 2003; Tronel \& Sara, 2002; Savic et al., 2000). The hippocampus, although it is implicated in some forms of olfactory memory, does not seem to be required for simple odor recognition tasks as used in the present experiments (Sauvage, Fortin, Owens, Yonelinas, \& Eichenbaum, 2008; Gottfried, Smith, Rugg, \& Dolan, 2004; Tronel \& Sara, 2003). Thus, a selective sensitivity of odor memory consolidation to manipulation of NA activity would also speak for a preferential impact of NA burst activity during SWS on amygdaladependent memories. It might be argued that the current data does not show a positive effect of sleep on declarative memory, and thus, clonidine could not affect sleep-related memory consolidation. However, previous studies showing an effect of sleep on word list memory used within-subject testing, because between-subject variance in memory performance is considerably greater than within-subject variance. Not finding differences between subject groups with such a small sample size cannot be interpreted as the absence of a sleep effect. Furthermore, the test for substance effects is done within-subject, with much greater statistical power. We can assume that a substance effect of relevant size would be detected in such a comparison.

A limitation of this and any human pharmacological study is that we cannot precisely determine the location of action of the substances administered. However, we do think that the data presented here make a strong case for a noradrenergic contribution to memory consolidation during sleep. Both substances used have different mechanisms of action, and both are independently and opposingly targeting the central noradrenergic system. Both substances are quite well known in their effects in the CNS. Because the LC is the only source of neocortical NA, we know that clonidine reduces general central noradrenergic availability by activating LC $\alpha 2$-autoreceptors. Likewise, reboxetine is known to be a selective noradrenergic reuptake inhibitor, thus increasing noradrenergic availability in limbic structures and the neocortex. It further may be argued in this context that the comparability of our two studies is limited because of the design differences between the experiments, particularly in sleep duration and the lengths of the retention interval, which were adapted to the different half-lives of the substances. Indeed, such differences prevent a direct comparison of absolute recall performance across both studies. However, both experiments included a placebo control condition, which was otherwise identical to the respective drug condition and was performed in the same subjects. It served as the primary reference for measuring the substance effects. Thus, our experiments must be interpreted as two independent experiments, which come to mutually supporting conclusions. Another limitation is that our experimental design cannot distinguish very well between different sleep stages, because the drugs were active throughout the whole sleep period. However, because there is no noradrenergic 
activity during REM sleep at all and administration of clonidine during wakefulness has no effect, it can be inferred that clonidine affects odor memory consolidation during non-REM sleep.

Reboxetine, apart from its use as an antidepressant, has been investigated mainly with regard to its modulation of working memory and its amygdala-dependent effects on emotional long-term memory (Chamberlain, Müller, Blackwell, Robbins, \& Sahakian, 2006). Reboxetine is a highly specific agent that blocks noradrenergic reuptake (Kent, 2000). It passes the blood-brain barrier and, at least in rodents, seems to be more available in the brain than in plasma after oral administration (Strolin Benedetti et al., 1995). When considering the absent peripheral changes in NA levels after reboxetine, other studies that measured plasma levels of NA after administration of a single dose of reboxetine are not known to the authors. However, a 3-day administration had no influence on plasma levels (Kelly, McAree, Cooper, \& Stevenson, 2002). On the other hand, increases in levels of plasma cortisol-as have been observed here-have been used as an indicator for elevated central norepinephrine levels, an effect that is mediated via increased release of corticotropin-releasing hormone in the paraventricular nucleus (Hill, Taylor, Harmer, \& Cowen, 2003). Therefore, we can be confident that reboxetine was active in our experiments, although peripheral levels did not follow its central action.

To summarize, the present results show that noradrenergic activity during sleep does not simply reflect lessened arousal but also has a functional significance in memory consolidation. Together with other recent findings, they show that there are multiple mechanisms acting on memory consolidation during sleep, depending on the memory systems involved (Born et al., 2006). Release of NA enhances odor memory consolidation only during non-REM sleep and, thus, must interact with other features of sleep. Therefore, it is our opinion that future studies of sleepdependent memory consolidation will have to consider sleep not as a unitary state but as the complex interaction of numerous changes in cortical and subcortical systems.

\section{Acknowledgments}

This work was supported by grants from Volkswagen Stiftung and DFG (SFB 654 "Plasticity and Sleep," Emmy-Noether Group "Memory Consolidation").

Reprint requests should be sent to Steffen Gais, Department of General and Experimental Psychology, Ludwig Maximilians University of Munich, Leopoldstr. 13, 80802 München, Germany, or via e-mail: gais@psy.lmu.de.

\section{REFERENCES}

Akirav, I., \& Richter-Levin, G. (2002). Mechanisms of amygdala modulation of hippocampal plasticity. Journal of Neuroscience, 22, 9912-9921.

Aston-Jones, G. (2004). The locus coeruleus, A5 and A7 noradrenergic cell groups. In G. Paxinos (Ed.), The rat nervous system (pp. 259-294). San Diego, CA: Elsevier Academic Press.

Aston-Jones, G., \& Bloom, F. E. (1981). Activity of norepinephrine-containing locus coeruleus neurons in behaving rats anticipates fluctuations in the sleep-waking cycle. Journal of Neuroscience, 1, 876-886.

Aston-Jones, G., \& Cohen, J. D. (2005). An integrative theory of locus coeruleus-norepinephrine function: Adaptive gain and optimal performance. Annual Review of Neuroscience, 28, 403-450.

Bear, M. F., \& Singer, W. (1986). Modulation of visual cortical plasticity by acetylcholine and noradrenaline. Nature, 320, $172-176$.

Berlau, D. J., \& McGaugh, J. L. (2006). Enhancement of extinction memory consolidation: The role of the noradrenergic and GABAergic systems within the basolateral amygdala. Neurobiology of Learning and Memory, 86, 123-132.

Berridge, C. W., \& Waterhouse, B. D. (2003). The locus coeruleus-noradrenergic system: Modulation of behavioral state and state-dependent cognitive processes. Brain Research Reviews, 42, 33-84.

Born, J., Rasch, B., \& Gais, S. (2006). Sleep to remember. Neuroscientist, 12, 410-424.

Bröcher, S., Artola, A., \& Singer, W. (1992). Agonists of cholinergic and noradrenergic receptors facilitate synergistically the induction of long-term potentiation in slices of rat visual cortex. Brain Research, 573, 27-36.

Buchanan, T. W., Tranel, D., \& Adolphs, R. (2003). A specific role for the human amygdala in olfactory memory. Learning and Memory, 10, 319-325.

Cahill, L., Pham, C. A., \& Setlow, B. (2000). Impaired memory consolidation in rats produced with beta-adrenergic blockade. Neurobiology of Learning and Memory, 74, 259-266.

Cahill, L., Prins, B., Weber, M., \& McGaugh, J. L. (1994). Beta-adrenergic activation and memory for emotional events. Nature, 371, 702-704

Chamberlain, S. R., Müller, U., Blackwell, A. D., Robbins, T. W., \& Sahakian, B. J. (2006). Noradrenergic modulation of working memory and emotional memory in humans. Psychopharmacology, 4, 397-407.

Cirelli, C., \& Tononi, G. (2000). Differential expression of plasticity-related genes in waking and sleep and their regulation by the noradrenergic system. Journal of Neuroscience, 20, 9187-9194.

de Quervain, D. J., Kolassa, I. T., Ertl, V., Onyut, P. L., Neuner, F., Elbert, T., et al. (2007). A deletion variant of the alpha2b-adrenoceptor is related to emotional memory in Europeans and Africans. Nature Neuroscience, 10, 1137-1139.

Dostert, P., Benedetti, M. S., \& Poggesi, I. (1997). Review of the pharmacokinetics and metabolism of reboxetine, a selective noradrenaline reuptake inhibitor. European Neuropsychopharmacology, 7(Suppl. 1), S23-S35.

Eschenko, O., \& Sara, S. J. (2008). Learning-dependent, transient increase of activity in noradrenergic neurons of locus coeruleus during slow wave sleep in the rat: Brain stem-cortex interplay for memory consolidation? Cerebral Cortex, 18, 2596-2603.

Foote, S. L., Bloom, F. E., \& Aston-Jones, G. (1983). Nucleus locus ceruleus: New evidence of anatomical and physiological specificity. Physiological Reviews, 63, 844-914.

Frank, M. G., \& Benington, J. H. (2006). The role of sleep in memory consolidation and brain plasticity: Dream or reality? Neuroscientist, 12, 477-488.

Frey, S., Bergado-Rosado, J., Seidenbecher, T., Pape, H. C., \& Frey, J. U. (2001). Reinforcement of early long-term 
potentiation (early-LTP) in dentate gyrus by stimulation of the basolateral amygdala: Heterosynaptic induction mechanisms of late-LTP. Journal of Neuroscience, 21, 3697-3703.

Gais, S., Albouy, G., Boly, M., Dang-Vu, T. T., Darsaud, A., Desseilles, M., et al. (2007). Sleep transforms the cerebral trace of declarative memories. Proceedings of the National Academy of Sciences, U.S.A., 104, 18778-18783.

Gais, S., Mölle, M., Helms, K., \& Born, J. (2002). Learning-dependent increases in sleep spindle density. Journal of Neuroscience, 22, 6830-6834.

Gibbs, M. E., \& Summers, R. J. (2002). Role of adrenoceptor subtypes in memory consolidation. Progress in Neurobiology, 67, 345-391.

Gold, P. E., \& van Buskirk, R. (1978). Posttraining brain norepinephrine concentrations: Correlation with retention performance of avoidance training and with peripheral epinephrine modulation of memory processing. Behavioral Biology, 23, 509-520.

Gottfried, J. A., Smith, A. P., Rugg, M. D., \& Dolan, R. J. (2004). Remembrance of odors past: Human olfactory cortex in cross-modal recognition memory. Neuron, 42, 687-695.

Graves, L., Pack, A., \& Abel, T. (2001). Sleep and memory: A molecular perspective. Trends in Neurosciences, 24, 237-243.

Harley, C. W. (2007). Norepinephrine and the dentate gyrus. Progress in Brain Research, 163, 299-318.

Hasselmo, M. E. (1999). Neuromodulation: Acetylcholine and memory consolidation. Trends in Cognitive Sciences, 3, 351-359.

Hill, S. A., Taylor, M. J., Harmer, C. J., \& Cowen, P. J. (2003). Acute reboxetine administration increases plasma and salivary cortisol. Journal of Psychopharmacology, 17, 273-275.

Hu, H., Real, E., Takamiya, K., Kang, M. G., Ledoux, J., Huganir, R. L., et al. (2007). Emotion enhances learning via norepinephrine regulation of AMPA-receptor trafficking. Cell, 131, 160-173.

Izquierdo, I., Medina, J. H., Izquierdo, L. A., Barros, D. M., de Souza, M. M., \& Mello e Souza, T. (1998). Shortand long-term memory are differentially regulated by monoaminergic systems in the rat brain. Neurobiology of Learning and Memory, 69, 219-224.

Ji, D., \& Wilson, M. A. (2007). Coordinated memory replay in the visual cortex and hippocampus during sleep. Nature Neuroscience, 10, 100-107.

Katsuki, H., Izumi, Y., \& Zorumski, C. F. (1997). Noradrenergic regulation of synaptic plasticity in the hippocampal CA1 region. Journal of Neurophysiology, 77, 3013-3020.

Kelly, C. B., McAree, S., Cooper, S. J., \& Stevenson, M. (2002). The effects of reboxetine, a noradrenaline reuptake inhibitor, on the plasma noradrenaline response to a cold pressor test in healthy volunteers. Journal of Psychopharmacology, 16, 333-336.

Kemp, A., \& Manahan-Vaughan, D. (2008). Beta-adrenoreceptors comprise a critical element in learning-facilitated long-term plasticity. Cerebral Cortex, 18, 1326-1334.

Kent, J. M. (2000). SNaRIs, NaSSAs, and NaRIs: New agents for the treatment of depression. Lancet, 335, 911-918.

Keranen, A., Nykanen, S., \& Taskinen, J. (1978). Pharmacokinetics and side-effects of clonidine. European Journal of Clinical Pharmacology, 13, 97-101.

Kindt, M., Soeter, M., \& Vervliet, B. (2009). Beyond extinction: Erasing human fear responses and preventing the return of fear. Nature Neuroscience, 12, 256-258.

Kirkwood, A., Rozas, C., Kirkwood, J., Perez, F., \& Bear, M. F. (1999). Modulation of long-term synaptic depression in visual cortex by acetylcholine and norepinephrine. Journal of Neuroscience, 19, 1599-1609.
Langer, S. Z. (1980). Presynaptic regulation of the release of catecholamines. Pharmacological Reviews, 32, 337-362.

Lestienne, R., Herve-Minvielle, A., Robinson, D., Briois, L., \& Sara, S. J. (1997). Slow oscillations as a probe of the dynamics of the locus coeruleus-frontal cortex interaction in anesthetized rats. Journal of Physiology Paris, 91, 273-284.

Llinas, R. R., \& Steriade, M. (2006). Bursting of thalamic neurons and states of vigilance. Journal of Neurophysiology, 95, 3297-3308.

Marshall, L., \& Born, J. (2007). The contribution of sleep to hippocampus-dependent memory consolidation. Trends in Cognitive Sciences, 11, 442-450.

Marshall, L., Helgadottir, H., Molle, M., \& Born, J. (2006). Boosting slow oscillations during sleep potentiates memory. Nature, 444, 610-613.

Mayers, A. G., \& Baldwin, D. S. (2005). Antidepressants and their effect on sleep. Human Psychopharmacology, 20, 533-559.

McGaugh, J. L. (2000). Memory-a century of consolidation. Science, 287, 248-251.

McGaugh, J. L. (2002). Memory consolidation and the amygdala: A systems perspective. Trends in Neurosciences, $25,456$.

McGaugh, J. L. (2004). The amygdala modulates the consolidation of memories of emotionally arousing experiences. Annual Review of Neuroscience, 27, 1-28.

McGaugh, J. L., \& Roozendaal, B. (2002). Role of adrenal stress hormones in forming lasting memories in the brain. Current Opinion in Neurobiology, 12, 205-210.

Miller, D. K., Wong, E. H., Chesnut, M. D., \& Dwoskin, L. P. (2002). Reboxetine: Functional inhibition of monoamine transporters and nicotinic acetylcholine receptors. Journal of Pharmacology and Experimental Therapeutics, 302, 687-695.

Mölle, M., Eschenko, O., Gais, S., Sara, S. J., \& Born, J. (2009). The influence of learning on sleep slow oscillations and associated spindles and ripples in humans and rats. European Journal of Neuroscience, 29, 1071-1081.

Mölle, M., Marshall, L., Gais, S., \& Born, J. (2002). Grouping of spindle activity during slow oscillations in human non-rapid eye movement sleep. Journal of Neuroscience, 22, 10941-10947.

Pace-Schott, E. F., \& Hobson, J. A. (2002). The neurobiology of sleep: Genetics, cellular physiology and subcortical networks. Nature Reviews Neuroscience, 3, 591-605.

Paré, D. (2003). Role of the basolateral amygdala in memory consolidation. Progress in Neurobiology, 70, 409-420.

Plailly, J., Bensafi, M., Pachot-Clouard, M., Delon-Martin, C., Kareken, D. A., Rouby, C., et al. (2005). Involvement of right piriform cortex in olfactory familiarity judgments. Neuroimage, 24, 1032-1041.

Plihal, W., \& Born, J. (1999). Memory consolidation in human sleep depends on inhibition of glucocorticoid release. NeuroReport, 10, 2741-2747.

Ramos, B. P., \& Arnsten, A. F. (2007). Adrenergic pharmacology and cognition: Focus on the prefrontal cortex. Pharmacology E Therapeutics, 113, 523-536.

Rasch, B., Dodt, C., Molle, M., \& Born, J. (2007). Sleep-stagespecific regulation of plasma catecholamine concentration. Psychoneuroendocrinology, 32, 884-891.

Rasch, B., Pommer, J., Diekelmann, S., \& Born, J. (2008). Pharmacological REM sleep suppression paradoxically improves rather than impairs skill memory. Nature Neuroscience, 12, 396-397.

Roozendaal, B., Hahn, E. L., Nathan, S. V., de Quervain, D. J., \& McGaugh, J. L. (2004). Glucocorticoid effects on memory retrieval require concurrent noradrenergic activity in the 
hippocampus and basolateral amygdala. Journal of Neuroscience, 24, 8161-8169.

Roozendaal, B., McEwen, B. S., \& Chattarji, S. (2009). Stress, memory and the amygdala. Nature Reviews Neuroscience, 10, 423-433.

Rosanova, M., \& Ulrich, D. (2005). Pattern-specific associative long-term potentiation induced by a sleep spindle-related spike train. Journal of Neuroscience, 25, 9398-9405.

Roullet, P., \& Sara, S. (1998). Consolidation of memory after its reactivation: Involvement of beta noradrenergic receptors in the late phase. Neural Plasticity, 6, 63-68.

Sanchez-Andrade, G., James, B. M., \& Kendrick, K. M. (2005). Neural encoding of olfactory recognition memory. Journal of Reproduction and Development, 51, 547-558.

Sara, S. J. (2009). The locus coeruleus and noradrenergic modulation of cognition. Nature Reviews Neuroscience, 10, 211-223.

Sara, S. J., Roullet, P., \& Przybyslawski, J. (1999). Consolidation of memory for odor-reward association: Beta-adrenergic receptor involvement in the late phase. Learning and Memory, 6, 88-96.

Sauvage, M. M., Fortin, N. J., Owens, C. B., Yonelinas, A. P., \& Eichenbaum, H. (2008). Recognition memory: Opposite effects of hippocampal damage on recollection and familiarity. Nature Neuroscience, 11, 16-18.

Savic, I., Gulyas, B., Larsson, M., \& Roland, P. (2000). Olfactory functions are mediated by parallel and hierarchical processing. Neuron, 26, 735-745.

Southwick, S. M., Davis, M., Horner, B., Cahill, L., Morgan, C. A., III, Gold, P. E., et al. (2002). Relationship of enhanced norepinephrine activity during memory consolidation to enhanced long-term memory in humans. American Journal of Psychiatry, 159, 1420-1422.

Stanton, P. K., \& Sarvey, J. M. (1985). Depletion of norepinephrine, but not serotonin, reduces long-term potentiation in the dentate gyrus of rat hippocampal slices. Journal of Neuroscience, 5, 2169-2176.

Steriade, M. (2003). The corticothalamic system in sleep. Frontiers in Bioscience, 8, d878-d899.

Strolin Benedetti, M., Frigerio, E., Tocchetti, P., Brianceshi, G., Castelli, M. G., Pellizzoni, C., et al. (1995). Stereoselective and species-dependent kinetics of reboxetine in mouse and rat. Chirality, 7, 285-289.
Sulmont, C., Issanchou, S., \& Köster, E. P. (2002). Selection of odorants for memory tests on the basis of familiarity, perceived complexity, pleasantness, similarity and identification. Chemical Senses, 27, 307-317.

Tononi, G., \& Cirelli, C. (2006). Sleep function and synaptic homeostasis. Sleep Medicine Reviews, 10, 49-62.

Tronel, S., Feenstra, M. G., \& Sara, S. J. (2004). Noradrenergic action in prefrontal cortex in the late stage of memory consolidation. Learning and Memory, 11, 453-458.

Tronel, S., \& Sara, S. J. (2002). Mapping of olfactory memory circuits: Region-specific c-fos activation after odor-reward associative learning or after its retrieval. Learning and Memory, 9, 105-111.

Tronel, S., \& Sara, S. J. (2003). Blockade of NMDA receptors in prelimbic cortex induces an enduring amnesia for odor-reward associative learning. Journal of Neuroscience, 23, 5472-5476.

Tully, K., Li, Y., Tsvetkov, E., \& Bolshakov, V. Y. (2007). Norepinephrine enables the induction of associative long-term potentiation at thalamo-amygdala synapses. Proceedings of the National Academy of Sciences, U.S.A., 104, 14146-14150.

Tzavara, E. T., Bymaster, F. P., Overshiner, C. D., Davis, R. J., Perry, K. W., Wolff, M., et al. (2006). Procholinergic and memory enhancing properties of the selective norepinephrine uptake inhibitor atomoxetine. Molecular Psychiatry, 11, 187-195.

van Stegeren, A. H., Wolf, O. T., Everaerd, W., \& Rombouts, S. A. (2008). Interaction of endogenous cortisol and noradrenaline in the human amygdala. Progress in Brain Research, 167, 263-268.

Vouimba, R. M., Yaniv, D., \& Richter-Levin, G. (2007). Glucocorticoid receptors and beta-adrenoceptors in basolateral amygdala modulate synaptic plasticity in hippocampal dentate gyrus, but not in area CA1. Neuropharmacology, 52, 244-252.

Washburn, M., \& Moises, H. C. (1989). Electrophysiological correlates of presynaptic alpha 2-receptor-mediated inhibition of norepinephrine release at locus coeruleus synapses in dentate gyrus. Journal of Neuroscience, 9, 2131-2140.

Williams, C. L., \& McGaugh, J. L. (1993). Reversible lesions of the nucleus of the solitary tract attenuate the memorymodulating effects of posttraining epinephrine. Behavioral Neuroscience, 107, 955-962. 